\title{
Retos y estrategias comunicacionales en la administración pública durante la emergencia sanitaria (COVID19)
}

\author{
Fredy Daniel Torres-Siavichay \\ fredy.torres@unl.edu.ec \\ https://orcid.org/0000-0001-5900-5998
}

Sybel Ontaneda-Andrade

sybel.ontaneda@unl.edu.ec

https://orcid.org/0000-0001-5947-6394

Eduardo Fabio Henríquez-Mendoza

eduardo.henriquez@unl.edu.ec

https://orcid.org/0000-0001-6102-9809

Hever Sánchez-Martínez

hever.sanchez@unl.edu.ec

https://orcid.org/0000-0001-5189-5164

Franklin Gustavo Santín-Picoita

franklin.santin@unl.edu.ec

https://orcid.org/0000-0002-3852-047X

Universidad Nacional de Loja

Loja - Ecuador

\section{RESUMEN}

Esta investigación centra su interés en analizar las estrategias comunicacionales externas que implementó la Dirección de Comunicación del Municipio de Loja para relacionarse con sus usuarios en el periodo más crítico de la pandemia. La metodología planteada se basa en determinar la efectividad y alcance de las comunicaciones mediante 387 encuestas realizada a los usuarios para conocer la opinión y aceptación ciudadana. Además, una la aplicación de fichas de observación a los comunicados emitidos en las principales redes sociales de la institución. Los resultados evidenciaron que existe mucho desconocimiento sobre las campañas comunicativas externas y una opinión regulardeficiente sobre los servicios comunicacionales brindados por la municipalidad. Por último, se realizó un análisis semiótico de dos artes que difundieron comunicados oficiales de carácter masivo. En conclusión, la mayor parte de la ciudadanía desconoce sobre las campañas comunicativas de la institución y se siente insatisfecha con la administración municipal.

Palabras clave: comunicación institucional; estrategias de comunicación; comunicación institucional externa; covid19 


\title{
Communications remains and strategies in the public administration during the health emergency (COVID-19)
}

\begin{abstract}
This research focuses its interest on analyzing the external communication strategies implemented by the Communication Directorate of the Municipality of Loja to interact with its users in the most critical period of the pandemic. The proposed methodology is based on determining the effectiveness and scope of communications through 387 surveys carried out with users to find out the opinion and public acceptance. In addition, join the application of observation files to the communications issued on the main social networks of the institution. The results showed that there is a lot of ignorance about external communication campaigns and a fair-deficient opinion about the communication services provided by the municipality. Finally, a semiotic analysis of two arts was carried out that disseminated official communiqués of a massive nature. In conclusion, most of the citizens are unaware of the institution's communication campaigns and feel dissatisfied with the municipal administration.
\end{abstract}

Keywords: institutional communication; communication strategies; external institutional communication; covid-19.

Artículo recibido: 15 noviembre. 2021 Aceptado para publicación: 10 diciembre 2021 Correspondencia: eduardo.henriquez@unl.edu.ec Conflictos de Interés: Ninguna que declarar 


\section{INTRODUCCIÓN}

Hoy en día, las redes sociales son parte fundamental en la vida de las personas y organizaciones, las cuales representan, un importante sistema de intercomunicación entre individuos y colectivos. Por ejemplo, las organizaciones utilizan este medio de comunicación para transmitir mensajes a gran cantidad de personas, de forma ágil y oportuna. También para visibilizarse ante lasociedad y comunicar todo aquello que aporte a la creación de una buena imagen institucional. Además, sirvenpara casos de emergencia como la provocada por la pandemia de la Covid-19 en la cual las redes socialesfueron un canal de comunicación y trabajo entre las organizaciones, funcionarios y usuarios, caso de estudio, los municipios que se acogieron al teletrabajo como el GADM de Loja.

Por ende, el presente trabajo expone una investigación realizada en el área de la comunicación institucional, donde se analizó las estrategias comunicacionales que utilizó el Municipio de Loja para dirigirse a sus usuarios mediante las redes sociales en los primeros seis meses de la pandemia. Por ello, su objetivo fue analizar las publicaciones para determinar la efectividad y el alcance de los comunicados masivos emitidos por las plataformas digitales institucionales. Fue así que, mediante la realización de encuestas se conoció la opinión y aceptación ciudadana ante la comunicación. Asimismo, las entrevistas realizadas a diferentes expertos, ayudaron a comprender la problemática y permitieron realizar un breve análisis semiótico de un diseño gráfico.

Para cumplir con el objetivo general se describe y analiza las estrategias de comunicación externa, las cuales se obtuvo a partir de la entrevista realizada a la directora de la Dirección de Comunicación del Municipio de Loja. Quien exclamó que las estrategias externas comunicativas empleadas en los primeros meses de la pandemia giraron en torno a tres campañas realizadas por la institución en el periodo de estudio, la primera denominada \#QuédateEnCasa, la segunda: Loja se reactiva segura \#NoBajesLaGuardia y la tercera: \#LojaSeCuida, realizadas en mediostradicionales y digitales. Estas campañas se cumplieron con la elaboración de varios vídeos o spots publicitarios, capsulas de audio y una batería de artes y diseños orientados a informar sobre las restricciones de movilidad, horarios de atención en mercados y en la institución. 
El objetivo de etas estrategias era llegar en aquel momento crucial, con información rápida y confiable. Por lo cual, una de las herramientas que permitió llegar de forma oportuna y permanente a los ciudadanos, fueron las redes sociales y el canal televisivo municipal "Canal Sur”. Estos instrumentos jugaron un papel primordial para difundir las medidas dictaminadas por el COE Cantonal. Así también,para difundir la campaña de comunicación a través de medios de comunicación ATL y BTL.

Los resultados determinaron que la Dirección de Comunicación del Municipio de Loja tiene un bajonivel de efectividad y alcance en sus productos comunicacionales difundidos en redes sociales, si se compara el número de habitantes existentes en el cantón Loja, con los ciudadanos que reaccionaron a los comunicados analizados. Por otra parte, e referencia a la opinión se conoció que la mayoría de la población desconoce sobre las campañas comunicativas de la institución y de las que conocen, la mayoría de las personas se sienten, algo satisfechas, con la comunicación establecida por la entidad, encuanto a la semiótica de la imagen implementada en los artes se identificó que si se emplean las normasbásicas de composición en los diseños, pero falta implementar símbolos o frases de la cultura lojana enlos artes para que el usuario se identifique con la institución y así se genere mayor familiaridad.

\section{Estrategias comunicacionales}

Desde la antigüedad hace aproximadamente 2.500 años, los chinos usaron el concepto de estrategia en asuntos de la milicia, luego en el año de 1960 las organizaciones empiezan a usar este concepto para reducir riesgos y potenciar oportunidades, así lo explica Humberto Serna Gómez en su libro titulado "Gerencia Estratégica". Por ello, este contexto histórico es posible definir a la estrategia una planificación que consiste en "fijar objetivos, acciones y recursos que orienten al desarrollo de una organización" (Serna, 2008, p.10). A su vez, el mexicano Miguel Aguado, teórico de la comunicación expone lo siguiente: "La comunicación estratégica es una interactividad, una hipótesis de trabajo para asistir en los procesos de comunicación que produzca una institución o cualquier sujeto social (2004, p. 230). Es una proyección compleja que desarrolla procesos comunicacionales, que pueden ser opuesto al mismo sistema mecanicistas y de experiencias inacabadas dentro de las mismas instituciones que aplican esta comunicación.

Así, de manera sistematizada y coherente, la imagen, marca y producto de cierta 
organización es capazde llegar de forma certera, a su público objetivo, con el propósito de convertirlos en clientes. En definitiva, estas estrategias deben ser bien elaboradas para garantizar el éxito de una comunicación externa. Por ende, hay que tener en cuenta la siguiente concepción semántica, según el autor HumbertoSerna, en su libro gerencia estratégica, la palabra estrategia significa "Definir lo que uno debe hacer y lo que no debe hacer" (1994, p. 15).

\section{Comunicación externa}

La comunicación como tal es un eje transversal dentro cualquier organización ya sea pública o privada. Esta comunicación, se refiere a que es la línea que cruza todos los departamentos y direcciones dentro de una organización. Por ello, el papel fundamental de la comunicación externa es velar por la imagen y el bienestar integral de la organizaciónen la sociedad y se Comunicación externa define como "el conjunto de operaciones de comunicación destinadas a los públicos externos de una empresa o institución" (Rosales, 2015, p. 20). Es decir, que la comunicación debe interrelacionarse con toda la estructura para gestionar las actividades planificadas que permitan cumplir los objetivos determinados.

\section{Efectividad de la Comunicación Institucional}

Para determinar la efectividad de la comunicación institucional es necesario conocer lo que sostiene el autor Francisco Giral (2017), el autor señala que el rol de la comunicación organizacional se centra en varios pilares de la cultura de la efectividad "porque el objetivo principal es iluminar y contagiar el propósito de la institución, logrando que se respire en la empresa la razón de existir que tiene la misma para que se comparta con gusto entre quienes la trabajan (pág. 1-6).

Bien sea, para cumplir con objetivo y función de la empresa y sus colaboradores, hay que generar una base de vinculación empática y de interacción. Esto garantizará el logró de los objetivos planteados.

\section{Alcance de la Comunicación Institucional}

El alcance de un proceso comunicacional, holísticamente, se refiere al nivel de persuasión, información, educación y entretenimiento que logra cumplir determinada persona o entidad frente a un gran número de personas. Ante ello, María Neme en su artículo, hace referencia a lo siguiente: "La base sobre la cual se fundamenta el mercadeo, es sin lugar a duda, la comunicación masiva. De hecho, el mercadeo es en sí 
mismo un sistema de comunicación general" (2015, p.1). El alcance se nutre básicamente de una planificación previa que contempla la delimitación de objetivosen un determinado tiempo y los campos de operatividad en los cuales se aplicaran las diferentes estrategias.

Para delimitar el alcance de un plan estratégico comunicacional y de las estrategias como tales, es necesario trazar objetivos que marquen un recorrido, para lo cual se necesita definir el presente y visualizar el futuro. Por ello el especialista en comunicación institucional sostiene que: "la materia primapara proyectar y diseñar los destinos de la organización, en gran medida se compone por nuestras competencias técnicas, por nuestros valores, creencias e ideologías y de nuestra percepción respecto a los cambios y oportunidades" (Manucci, 2004, p. 4-21). En definitiva, el alcance comprende el nivel de persuasión que ejerce una estrategia de comunicaciónsobre la conducta u opinión de una o más personas que buscan una interacción en específico.

\section{Semántica y semiología en torno a la Comunicación institucional}

El término semántica fue acuñado por Michael Bréal en el año de 1833. Se denomina semántica a la ciencia lingüística que estudia el significado de las palabras y expresiones, es decir, lo que las palabras quieren decir cuando hablamos o escribimos. En este sentido, la semántica tiene como finalidad descomponer el significado en unidades más pequeñas, denominadas semas o rasgos semánticos, los cuales sirven para segmentar el significado de las palabras, y diferenciar palabras de significado parecidoy palabras de significado opuesto (Definiciones de Oxford Language, 2020, p. 1).

Por ello, el semiólogo y escritor italiano Umberto Eco (1932-2016) problematiza la noción de semántica, entendida de manera sintetizada como: "el estudio del significado de las palabras" (Eco, citado en Acta Poética, 2018, p.1). Asimismo, el reconocido escritor de la famosa novela denominada "En el nombre de la Rosa", sustentalo siguiente: "las concepciones de la semántica pertenecen a diversas escuelas de la lingüística, la filosofía de lenguaje, la teoría literaria y la lexicografía para poner de manifiesto la complejidad del término y, por tanto, la idea de sentido" (Eco, citado en Acta Poética, 2018, p.1).

\section{Redes sociales y contenidos digitales}

En la actualidad las instituciones públicas mediante sus Direcciones de Comunicación buscan potenciar la interacción comunicacional con sus públicos externos mediante las 
plataformas digitales que permiten establecer una comunicación integral y de doble vía con sus usuarios. Con este antecedente traemos a colación lo que dice el siguiente razonamiento: "Lo que da vida a las redes socialesson las interacciones con los autores y las personas que reaccionan al comunicado, más no, la tecnología que representa un canal de comunicación" (Suasnabas, 2018, p. 57). Por ende, las empresas buscan crear una buena imagen empleando buenos contenidos informativos que permiten tener buena aceptación.

Algo de mucha importancia hoy en día es el feedback o retroalimentación de la institución pública con su público externo objetivo. Por ello, se debe tener en cuenta tres aspectos básicos como son: conexión con su público, mensaje claro y preciso, y cuidar la imagen de la Institución. A partir de estoselementos, se puede lograr que "los usuarios compartan, comenten y etiqueten generando un tráfico orgánico que aumente la interacción entre instituciones y usuarios" (Orellano, 2020, p.127).

\section{Redes sociales}

De forma general, las redes sociales son un conjunto de comunidades formadas en redes virtuales interconectadas que existen en el ciberespacio. Estas redes son usadas por usuarios y organizaciones queestablecen alguna relación entre sí, mediante el uso de estas plataformas en internet. Las redes sociales de más consumo en la ciudadanía lojana son el Facebook, Twitter e Instagram, según un estudio realizadopor la consultora de mercadeo Qbit_Agencia_Digital (2020).

Por otra parte, estos espacios de interacción social permiten a las personas asociarse en grupos e intercambiar información, mediante elementos de comunicación como imágenes, vídeos, textos, entre otros que dependen del interés y la necesidad del usuario. Cada red social tiene sus objetivos y usos particulares, su naturaleza se adapta al contenido que se busca difundir ante miles de personas por el mundo entero. Por ejemplo, Facebook es una red social muy útil para contactar amigos, conocer gente con los mismos intereses y crear páginas de empresas. Esto último para mejorar el branding yposicionamiento (Briciu, V. A. et al., 2021).

Dado que, Twitter es una plataforma de microblogging con mensajes que regulan un número máximo de caracteres. Así, esta red sirve principalmente para transmitir información y opiniones. Por último, "Instagram es una red social que tiene como propósito principal compartir fotografías que son puestas ante una variedad de filtros que 
alteran su estética, esta red social se conecta con otras para difundir la información" (Ramos, 2015, p. 1). Esta aplicación también sirve para empresas que buscan nuevas formas de Marketing para llegar a sus públicos de interés debido al gran impacto visual que causan.

\section{Gestión y producción de contenidos para redes sociales}

En la actualidad las redes sociales han revolucionado la vida de las personas y forman parte de su diario vivir. Por ende, para el experto en el tema Toledano (2015), estos espacios digitales de intercambio social constituyen un medio de comunicación alternativo, bastante eficaz para difundir contenidos de variada y diversa naturaleza con amplio alcance, buscando conectar audiencias cada vez más fragmentadas, por ello, "resulta necesario diversificar los contenidos e innovar en los mismos, creando espacios de interrelación bastante participativos con nuestros públicos” (p. 4).

Si se analiza lo anterior, es posible conocer que el secreto para crear contenidos de calidad, atractivos y amigables con las audiencias deben ser creativos; innovadores y serviciales con las necesidades de nuestros consumidores finales. "El contenido de calidad puede rápidamente convertirse en el alma de la institución al interior del mundo en línea" (Handley, 2012, p. 11). En este sentido, los Millennials son usuarios intensivos de Redes Sociales. Según Ruiz (2017), “hay más de 2.700 millones de personas en el mundo con presencia en Redes Sociales y sólo Facebook cuenta con 1.700 millones de usuarios activos mensuales" (p. 10).

Por otra parte, para cumplir con nuestro objetivo es necesario planificar estrategias comunicativas queutilicen las nuevas tecnologías como soporte, porque hoy en día "los consumidores buscan marcas que les aporten algo más, en forma de experiencia, entretenimiento, formación, información e incluso que les aporten soluciones útiles en su vida" (García, 2009, p.4). Por efecto, "si las organizaciones son innovadoras y relevantes para las personas, entonces los individuos hablarán de la compañía, a través del auge de las redes sociales y de los medios ganados que harán la comunicación de marca, contarán suhistoria” (Montague, 2013, p.1).

Asimismo, Cuervas indica que "el reto de las empresas es definir la clase de contenidos pueden generar para marcar una diferencia con la competencia. Además, encontrar un mecanismo que permita atraer y enganchar a sus públicos" (Cuervas, 2015, p. 37). 


\section{Redes sociales en instituciones públicas.}

Uno de los principales activos que tiene mucho valor tanto en las organizaciones privadas como en las públicas es la información y el posicionamiento de su imagen, marca en la mentalidad de las personas y por ende, en el mercado. Es así, que las redes sociales constituyen la herramienta de vital importanciaque permiten visibilizar y comunicar adecuadamente la cultura organizacional, actividad y naturaleza de la institución hacia sus usuarios, clientes o públicos de relación, para que las personas la perciban dela forma que pretenden los estrategas comunicadores de cada institución. Esto sucede todo, en torno a los objetivos yrazón de ser de la empresa u organización, es por ello que: "para que una institución pública tenga presencia, influencia y notoriedad en las redes sociales, debe divulgar en ellas, información verdadera ypertinente para la ciudadanía" (Cueva, 2018, p.1).

En este sentido, existen diferentes estrategias digitales que aportan a mejorar el posicionamiento de la organización en redes sociales como la observación y el software bakers Social, como un instrumentode análisis digital computarizado. Ante esta realidad hoy en día las instituciones públicas como los municipios, prefecturas, ministerios y GADS parroquiales cuentan con personal encargado de manejar y administrar las redes sociales para comunicar e informar correctamente a sus ciudadanos de manera ágily oportuna todas y cada una las actividades que llevan a cabo. Lo que buscan es el bienestar de sus comunidades, normas e información para sus diferentes públicos que se encuentran en las redes sociales, sobre todo, en estos tiempos de crisis sanitaria, social y económica, donde las redes son herramientas de comercio y difusión de contenidos gratuitas que permiten generar una comunicación cercana y de doble vía conlos usuarios o público específico.

\section{Comunicación en Crisis Sanitaria}

La pandemia desatada a partir de la propagación del virus mortal SARS-CoV-2 genera fuertes crisisen distintos aspectos como la salud, economía, sociedad y en las distintas áreas donde se desarrollan las diferentes actividades humanas, entre ellas, la Comunicación en un contexto de falta de información veraz y de noticias falsas en torno a la Covid-19. Por ello, la Organización Mundial de la Salud en el marco del Sixth Futures Forum on Crisis Communication (2004) recoge: "En salud, crisis y comunicación están íntimamente relacionadas. Todas las crisis de salud son también 
crisis de comunicación”. El papel que cumple la comunicación consiste en explicar lo que sucede en la realidad del País y en torno a la pandemia, sobre todo en lo relacionado con el riesgo, la prevención y los efectosen la salud del Coronavirus.

En este aspecto se reconoce la existencia de incertidumbre en la sociedad que se trata de un virus nuevo del cual se desconoce su naturalidad y forma de afección, ya que los síntomas son bastante relativos, así lo sostiene la Organización Mundial de la Salud (OMS) cuando expresa que "la ciencia reconoce cierto grado de incertidumbre sobre muchos riesgos y la magnitudde otros no se conoce en profundidad, como la Covid-19. Además, no todas las personas afrontan el mismo nivel de riesgo, que puede estar desigualmente distribuido entre la población" (OMS, 2020). Por otra parte, en una entrevista realizada a un experto en Comunicación estratégica, se destaca la importancia de semaforizar el estado de esta, parapoder reaccionar adecuadamente ante las diferentes realidades que afectan a la imagen pública de loa institución.

\section{ESTRATEGIAS METODOLÓGICAS O MATERIALES Y MÉTODOS}

Para esta investigación, de corte cuantitativa-cualitativa, se utilizó el proceso deductivo, es decir, se inició con una revisión bibliográfica, que ayudó a razonar y explicar la realidad del objeto de estudio. Luego se partió de teorías generales hacia casos particulares. Algunos autores plantean ciertas incertidumbres de cara relación entre las partes cualitativa y cuantitativas involucradas en este tipo de investigación (Akerblad et al., 2020; Uprichard y Dawney, 2016). Pero, de acuerdo con Creswell y Plano-Clark, quienes justifican la pertinencia de esta mixtura, esta investigación se eligió integrar estos dos enfoques para dar respuesta integral a las variables principales planteadas (2018). El universo investigado y del cual se tomó la muestra, es la población económicamente activa del cantón Loja, misma que abarca un total de 42.883 personas, según el censo poblacional del 2010 (INEC). Como criterio de selección para nuestra muestra se utilizaron tres técnicas: la primera fue una fórmula matemática que permitió cuantificar la población. Por ello, se aplicó una encuesta a 387 personas. Con esta técnica permitió conocer la opinión ciudadana y aceptación del usuario hacia la comunicación querealiza la entidad. La segunda técnica, fueron las fichas de observación; mismas que posibilitaron conocer el nivel de efectividad y alcance o difusión de las estrategias comunicativas externas aplicadas por la Dirección de Comunicación del municipio de Loja desde marzo hasta septiembre del 2020, donde se evidenció el nivel máximo 
posible de reacciones que alcanzaron las de mayor interactividad. La tercera técnica, fue realizar una serie de entrevistas que se aplicaron a cincoprofesionales en el campo de esta tesis (Rodríguez Jiménez, et al., 2017).

\section{RESULTADOS Y DISCUSIÓN}

Para recabar la opinión de la ciudadanía lojana, sobre el objetivo central del presente estudio, se aplicó una encuesta a una muestra de 387 personas de ambos sexos, con diversas edades. Se empleó la herramienta de Google Formularios. Para efectos de esta exposición de resultados y en atención a lascaracterísticas del informe se incluyen aquellas preguntas que se consideran más relevantes para verificar los objetivos propuestos en el presente artículo.

¿Usted se informó de manera clara sobre las actividades que desarrolló el Municipio deLoja durante la emergencia sanitaria?

Tabla 1. Información de actividades.

\begin{tabular}{|l|c|c|}
\hline \multicolumn{1}{|c|}{ Variables } & Frecuencia & Porcentaje (\%) \\
\hline $\mathrm{Si}$ & 73 & $19 \%$ \\
\hline Un poco & 216 & $56 \%$ \\
\hline $\mathrm{No}$ & 98 & $25 \%$ \\
\hline Total & 387 & $100 \%$ \\
\hline
\end{tabular}

Elaboración propia (2021).

Gráfico 1. Información de actividades

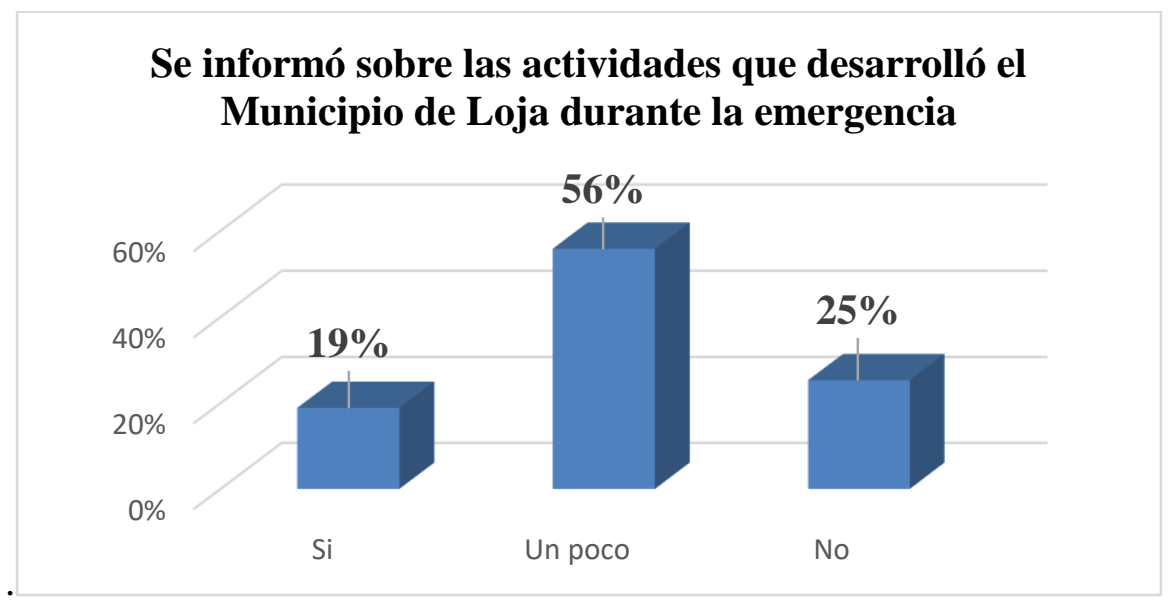

Elaboración propia (2021).

\section{Análisis cuantitativo}

De la totalidad de encuestados el $56 \%$ que corresponde a 216 personas se informó un poco sobre las actividades que desarrolló el Municipio de Loja en la pandemia, asimismo 
un $25 \%$ no se informó que corresponde a 98 personas, frente a un $19 \%$ que si se informó; cifra que representa a 73 personas consultadas.

\section{Análisis cualitativo.}

En los resultados de esta pregunta se evidencia que, de todas las personas encuestadas, tan solo un $19 \%$ se informó de los comunicados emitidos por el Municipio de Loja en los seis primeros meses de la pandemia. Esto se da, porque siguen las redes sociales y les interesa conocer lo que sucede en la administración dela ciudad, frente al $25 \%$ que no lo hizo porque no sigue las redes sociales y pasan ocupados en otras actividades. Por otra parte, la mayoría de las personas consultadas se informaron, un poco, representadasen un $56 \%$; ya que de repente vieron algo del tema en alguna red social de su preferencia como Facebook.

¿Indique el nivel de satisfacción que tiene usted hacia los servicios comunicativos prestados porsu Municipio?

Tabla 2. Servicios comunitarios.

\begin{tabular}{|l|c|c|}
\hline \multicolumn{1}{|c|}{ Variables } & Frecuencia & Porcentaje \\
\hline Muy satisfecho & 25 & $6 \%$ \\
\hline Bastante satisfecho & 62 & $16 \%$ \\
\hline Algo satisfecho & 230 & $59 \%$ \\
\hline Poco satisfecho & 57 & $15 \%$ \\
\hline Nada satisfecho & 13 & $3 \%$ \\
\hline Total & 387 & $100 \%$ \\
\hline
\end{tabular}

Gráfico 2. Servicios comunitarios

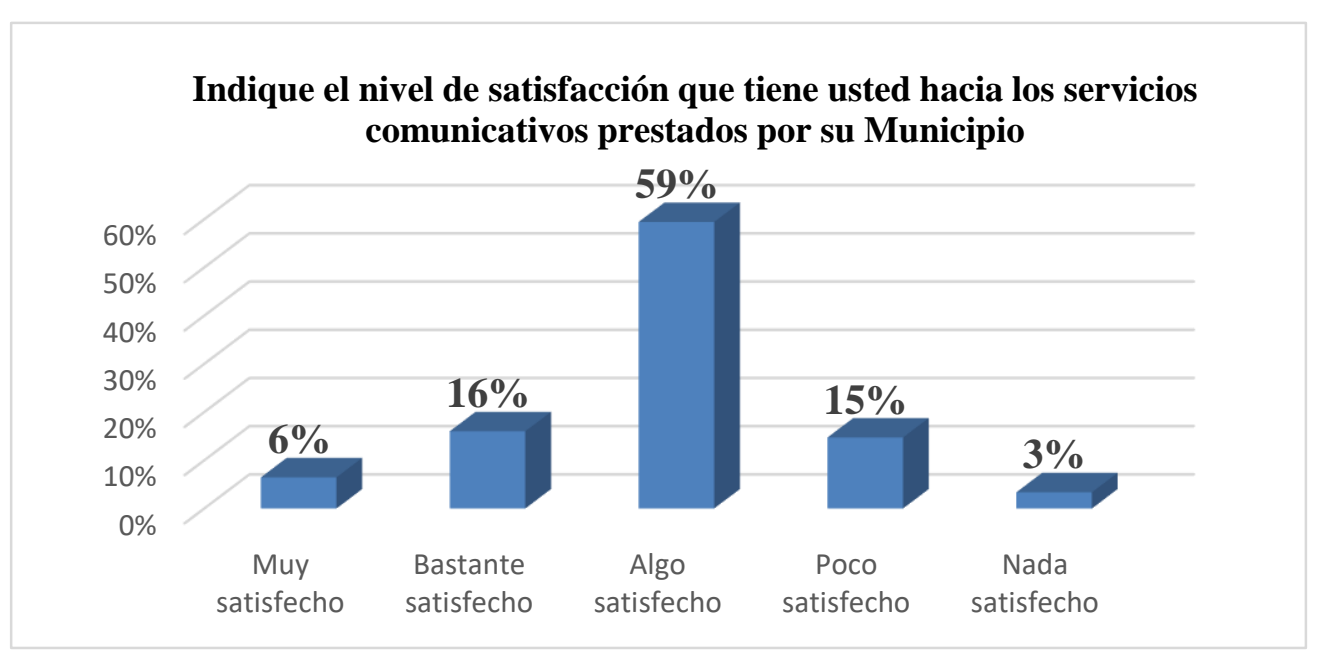

Elaboración propia (2021). 


\section{Análisis Cuantitativo}

Esta pregunta aporta a desarrollar los objetivos específicos que buscan determinar la efectividad y el alcance de las estrategias comunicacionales externas y también a conocer la opinión de la ciudadanía, frente a las mismas. En esta pregunta 248 personas que representan un $64 \%$ de las 387 encuestadas respondieron que no conocen sobre alguna campaña comunicativa que realiza el Municipio de Loja, Mediante la Dirección de Comunicación y 139 respondieron que si conocen sobre alguna campaña desarrollada por la municipalidad; esta parte de la población encuestada representa un $36 \%$.

\section{Análisis Cualitativo}

Ante esta interrogante la mayoría de personas aglutinadas en un $64 \%$ respondieron que no conocensobre ninguna campaña que realice el Municipio de Loja porque no ven nada en redes sociales u otros medios comunicacionales de amplio alcance y tan solo un $36 \%$ de los encuestados conoce sobre algunacampaña comunicativa que realiza la institución pública con su Dirección de Comunicación, porque pormotivos de trámites e información oficial siguen las redes sociales de la institución. Es decir, que pocaspersonas conocen sobre las campañas comunicativas que se realiza el Municipio de Loja para prevenir la infección masiva del Covid-19 y comunicar las disposiciones y restricciones oficiales del Municipio de Loja, para evitar la circulación de información falsa, confusiones y un desorden de caráctercomunicativo.

¿Cuál es la red social que prefiere usted para informarse sobre las noticias, acciones y comunicados que emite el Municipio a la ciudadanía?

Tabla 3. Red social de preferencia.

\begin{tabular}{|l|c|c|}
\hline \multicolumn{1}{|c|}{ Variables } & Frecuencia & Porcentaje \\
\hline Facebook & 287 & $74 \%$ \\
\hline Twitter & 26 & $7 \%$ \\
\hline Instagram & 74 & $19 \%$ \\
\hline Total & 387 & $100 \%$ \\
\hline
\end{tabular}

Elaboración propia (2021). 
Gráfico 3. Red social de preferencia.

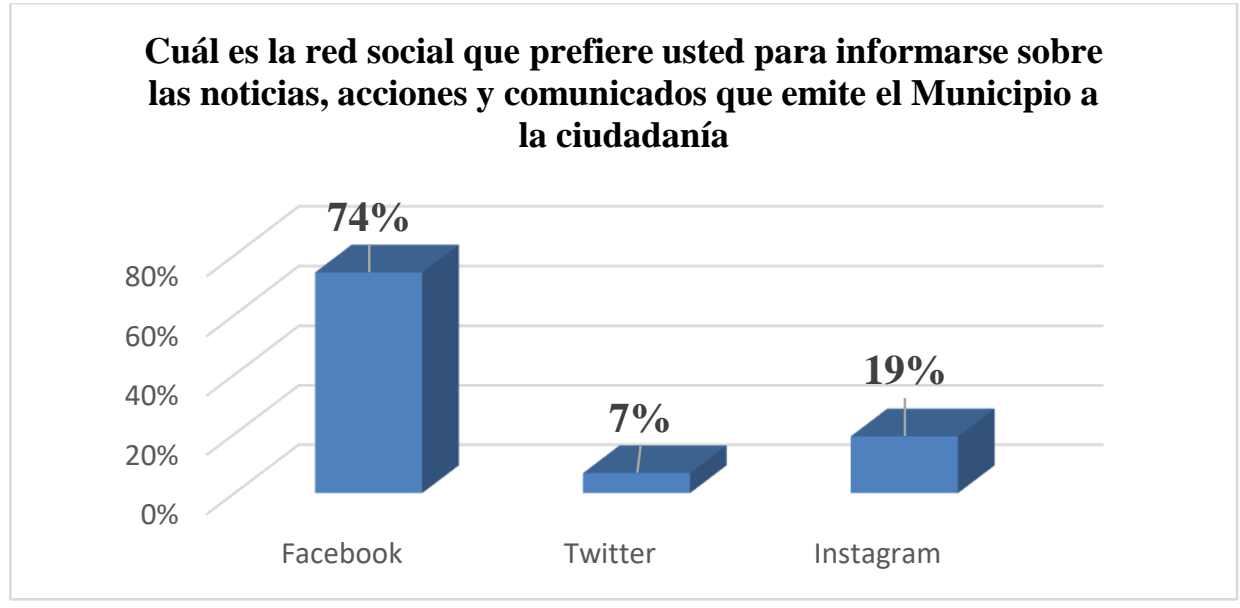

Elaboración propia (2021).

\section{Análisis cuantitativo}

De las 387 personas encuestadas 287 prefiere informarse sobre las actividades que desarrolla en Municipio de Loja por Facebook, cantidad que representa un $74 \%$. La segunda red social que prefieren es Instagram con un $19 \%$ que abarca a 74 personas. Por último, un $7 \%$ representado en 26 personas prefieren que se comuniquen con ellos por Twitter.

\section{Análisis cualitativo}

Los resultados revelan que la mayoría de las personas desean ser informadas por Facebook; ya que un 74\% que comprende a 287 encuestados respondieron que prefieren informarse por esta red social. La red social es de fácil acceso y la más popular, mientras que la minoría encuestada; prefiere ser informada a través Twitter, estas representan un $7 \%$ del total, que comprende a 26 personas, debido a qué esta plataforma es su fuente información por la confiabilidad y credibilidad del caso. Al tanto que, el 19 por ciento prefiere informarse por Instagram, cantidad que abarca a 74 personas, especialmente un público joven, quienes en su mayoría son estudiantes. Todo esto, debido al uso y preferencia que tiene la ciudadanía con cada red social y la importancia brindada a los contenidos municipales. 
¿Piensa que el Municipio de Loja realiza de manera eficiente la comunicación hacia sus usuarios?

Tabla 3. Eficiencia del Municipio

\begin{tabular}{|l|c|c|}
\hline Variables & Frecuencia & Porcentaje \\
\hline si & 201 & $52 \%$ \\
\hline No & 186 & $48 \%$ \\
\hline Total & 387 & $100 \%$ \\
\hline
\end{tabular}

Elaboración propia (2021).

\section{Gráfico 3.}

Eficiencia del Municipio.

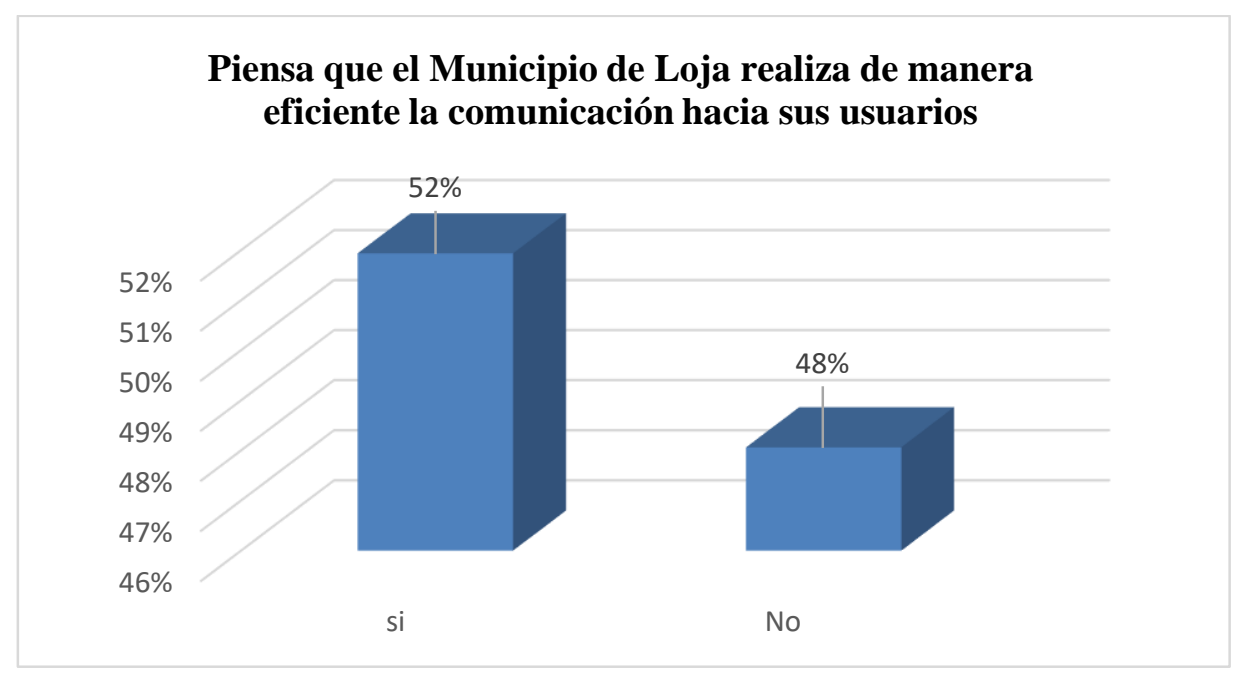

Elaboración propia (2021).

\section{Análisis Cuantitativo}

Comenzamos nombrado que 201 personas contestaron que el Municipio de Loja si realiza una comunicación eficiente con sus usuarios; cantidad que constituye un $52 \%$ de los 387 encuestados. Y, 186 que representan un $48 \%$ de los encuestados consideran que el Municipio no realiza de manera eficiente la comunicación con la ciudadanía.

\section{Análisis Cualitativo}

En esta pregunta la mayoría de las personas contemplada en un $52 \%$ del total de encuestados piensa que el Municipio de Loja realiza de manera eficiente la comunicación con sus usuarios. Porque conoce sobre el trabajo que se realiza, pero la diferencia es poca, ante un $48 \%$ que considera que el Municipio de Loja, no realiza una comunicación eficiente. Lo que quiere decir que un amplio sector de la sociedad piensa que no se 
cumplen los objetivos comunicativos, usando pocos instrumentos, por parte de quienes trabajan en la Comunicación Organizacional externa del Municipio de Loja.

\section{Discusión}

Se determinó la Opinión Ciudadana frente a las estrategias de comunicación externa implementadas por la Dirección de Comunicación del Municipio de Loja en tres campañas comunicacionales que buscaban combatir la propagación de la Covid-19 en la ciudad. de Loja, realizando 387 encuestas donde se destacó que el $64.1 \%$ de usuarios, no conoce sobre las campañas comunicativas que se realizaron en el Municipio de Loja, frente a un $35.9 \%$ que conoce sobre las campañas, por otro lado un $55.8 \%$ de los encuestados se informó, un poco; de manera clara, sobre las actividades que desarrolló el Municipio de Loja durante la emergencia sanitaria, mientras un $25.3 \%$ expresó que, no, se informó de nada; frente a un 18.9 \% que afirmó, si, conocer sobre los comunicados oficiales del Municipio. Por otra parte, un $49.1 \%$ de la población encuestada considera que el Municipio de Loja, no, mantiene una comunicación correcta con el usuario. Asimismo, un $59.4 \%$ se siente algo satisfecho con los servicios brindados por el Municipio de Loja.

En este sentido, cabe destacar que el Municipio de Loja tiene un nivel bajo de efectividad y alcance en sus estrategias de comunicación externa; difundidas en sus redes sociales oficiales, porque, la mayoría de la opinión ciudadana desconoce sobre las campañas comunicativas que realiza la Dirección de Comunicación del Municipio de Loja, por falta de difusión de estas; en medios con mayor audiencia en la ciudad de Loja. Por otra parte, las personas que conocen sobre esta información se sienten algo satisfechas de su calidad, finalmente se destaca que, en la Dirección de Comunicación se emplea adecuadamente la semántica en referencia a la teoría de la imagen que se refiere a la semiótica de la imagen; pero si le falta innovar y manejar de mejor forma la utilización de los símbolos en sus artes, para que las personas se identifiquen y familiaricen con los procesos de comunicación externa.

La actual página oficial de Facebook que usa el Municipio de Loja se crea en el año 2014 y cuenta con 107 mil seguidores, hoy en día es la red social o plataforma digital más utilizada por las personas que habitan el cantón Loja y a la vez, constituye el medio por el cual los usuarios del Municipio prefieren recibir los diferentes comunicados generados por la institución, esta afirmación se realiza con base en los datos recolectados al realizar 
la encuesta que determinó con un $69.9 \%$ el hecho de que el Municipio debe usar Facebook para entablar una comunicación efectiva, clara y oportuna con sus usuarios.

Al revisar las reacciones de los usuarios con la comunicación trazada por el Municipio de Loja en la red social Twitter se identificó que el mayor nivel de alcance y efectividad evidente en las reacciones representó un total de 481 en un tweet que pertenece a un spot, dentro de la campaña \#LojaSeCuida, cantidad que es mínima ante el número de personas que habitan el cantón Loja. Por esta razón el nivel de efectividad y alcance de la comunicación externa es de bajo nivel, debido a que las personas eligen esta red social en un $4.7 \%$ como canal de su preferencia para que el Municipio establezca una comunicación directa con ellos, lo cual se evidencia en la encuesta aplicada.

El nombre de la cuenta oficial de Instagram que usa el Municipio de Loja es @ municipiodeloja; en la actualidad cuenta con 25.6 mil seguidores y reúne un total de 6059 publicaciones. Este medio de difusión sirve para llegar a un público joven, ya que esta clase de audiencia son quienes generan la comunicación de retorno, las reacciones.

En la investigación del número de reacciones presentes en la red social de Instagram que usa el Municipio de Loja se observa que el mayor número de personas que reaccionaron al comunicado fue de 109; en una publicación realizada el 14 de septiembre del 2020, la cual informa sobre las medidas de restricción vehicular dispuestas por el COE cantonal, ante la emergencia sanitaria. Esta cantidad es mínima frente a los más 200 mil habitantes que tiene el cantón, por este motivo se determina que en la comunicación realizada por la DIRCOM de Loja en Instagram tiene un bajo nivel de efectividad y un mínimo alcance.

\section{CONCLUSIÓN O CONSIDERACIONES FINALES}

Se puede concluir que las estrategias comunicativas externas implementadas por la Dirección de Comunicación del Municipio de Loja; mediante tres campañas difundidas en medios tradicionales y digitales, no son muy conocidas por una parte considerable de la población que vive en la ciudad de Loja. En este sentido, la efectividad y el alcance de los productos comunicativos que integran la campaña tienen un nivel bajo, si se considera a los 214.855 habitantes del cantón Loja; entendidas como el público objetivo de la institución pública.

Asimismo, después de tabular y analizar la encuesta aplicada se puede afirmar que la opinión y el nivelde satisfacción que tiene la ciudadanía ante la comunicación que se establece desde la Dirección de Comunicación del Municipio de Loja, con ellos; es bajo, 
porque en las encuestas aplicadas, la mayor parte de la ciudadanía desconoce sobre las campañas comunicativas de la institución y se siente algo satisfecha con aquello que logra conocer de la institución en cuestión.

Por otra parte, luego de comparar los conceptos de semántica con dos comunicados gráficos digitales realizados por la Dirección de Comunicación del Municipio de Loja, se concluye que en la municipalidad se utiliza regularmente la semántica para elaborar un arte y difundirlo en redes sociales ya que se considera las normas técnicas y los conceptos de la semántica que permiten generar impactoy establecer una comunicación efectiva con el usuario, lo cual no cubre la falta de la utilización de símbolos en el proceso de comunicación masiva, acorde la cultura del usuario y su contexto. Asimismo, falta innovar para comunicar más y mejor mediante redes sociales como Facebook, ya que el $74 \%$ de lapoblación encuestada prefiere esta red social para recibir información oficial del Municipio de Loja.

\section{LISTA DE REFERENCIAS}

Aguado, M. (2004). Introducción a las teorías de la información y comunicación. Depósito deinvestigación Universidad de Murcia.

AlBriciu, V. A., \& Briciu, A. (2021). Social Media and Organizational Communication. In Encyclopedia of Organizational Knowledge, Administration, and Technology (pp. 2609-2624). IGI Global.

Cueva, V. E., Benites Suquitana, J. L., Fuentes Díaz, A. I., \& Largo Valdiviezo, R. A. (2018). Elposicionamiento en redes sociales de las Instituciones Públicas del Ecuador. REVISTA CIENTÍFICA ECOCIENCIA, 5, 1-23. https://doi.org/10.21855/ecociencia.50.166

Cuervas-Mons, V., de la Rosa, G., Pardo, F., San Juan, F., \& Valdivieso, A. (2015). Actividad y resultados del trasplante hepático durante el período 1984-2012. Análisis del Registro Español de Trasplante Hepático. Medicina Clínica, 144(8), 337-347.

García. L. (2020). Comunicación emergente en el ámbito Institucional y Político. Depósito deinvestigación Universidad de Sevilla. Barcelona. IDUS.

Giral. F, et al. (25 de 08 de 2017). Cultura de la EFECTIVIDAD 2.0. Editorial LID, México. Obtenido de https://books.google.com.ec/books?id=8JEyDwAAQBAJ\&dq=efectividad+de+ 
$\underline{1 a+c}$

Bréal, M., Desmet, P., \& Swiggers, P. (1995). De la grammaire comparée à la sémantique: textes deMichel Bréal publiés entre 1864 et 1898 (Vol. 4). Peeters Publishers.

Manucci, M. (2004). Comunicación Corporativa estratégica. De la persuasión a la creación derealidades compartidas. Norma. Bogotá

Manucci, M. (2006). La estrategia de los cuatro círculos. Bogotá. Norma.

Manucci, M. (03-2005). Narraciones corporativas. Comunicación, estrategia y futuro en lasorganizaciones Razón y Palabra, núm. 43, febrero-marzo, 2005 Universidad de los Hemisferios Quito, Ecuador. Disponible en https://www.redalyc.org/articulo.oa?id=199520626019.

OMS. (2020). Preguntas y respuestas sobre el Coronavirus. Obtenido de https://www.who.int/es/emergencies/diseases/novel-coronavirus-2019

Orellano, S. (2020). Las redes sociales en la promoción turística. Estudio de caso: Catedral AltaPatagonia SA, Bariloche, Argentina.

Rosales Prado, M. M. (2015). La expansión internacional de las empresas ecuatorianas productoras debienes de consumo a través de la implementación de un plan de relaciones públicas (RRPP), caso aplicado al sector textil (Master's thesis, Universidad de Guayaquil Facultad de Ciencias Económicas).

Rosales. (22 de 07 de 2019). ¿Cómo desarrollar una estrategia de Comunicación?. Blog. RockContent. Disponible en https://rockcontent.com/es/blog/estrategias-decomunicacion/

Rodríguez Jiménez, Andrés, \& Pérez Jacinto, Alipio Omar (2017). Métodos científicos de indagación y

de construcción del conocimiento. Revista Escuela de Administración de Negocios, (82),1-26.

ISSN: 0120-8160. https://www.redalyc.org/articulo.oa?id=20652069006

Ruiz, J. (2017). Millennials y redes sociales: estrategias para una comunicación de marca efectiva. Journal 8 comunicación.

Serna. H. (15 de 08 de 2010). Gerencia Estratégica, teoría, metodología, alineamiento, implementación, mapas estratégicos, índices de gestión. 3R Editores edición 10. Bogotá. Disponible el documento escaneado en file://C:/Users/USUARIO/Downloads/Libro-gerencia- estrategica-humberto- 
sern.pdf

Serna, H. (1994). Gerencia Estratégica. Bogotá: Panamericana, 3R EDITORES.

Suasnabas, L. Chancusing, J. Carvajal, C. Constante, J. y Quinotoa, E. (2018). La Web 2.0, Redes Sociales y la Educación Superior. (ed., $1^{\circ}$ ). Mawil publicaciones deEcuador. Ure, M. (2016). La comunicación de la administración pública en las redes sociales: los casos de las ciudades de Buenos Aires y Bolonia. Palabra Clave, 19(1), 240-270.

Toledano. (25 de 08 de 2015). Herramientas de contenido en internet. Opción, 31 (4), 978-996.

Universidad del Zulia Maracaibo, Venezuela

Disponible

en:

ww.redalyc.org/articulo.oa?id=310/310455 